VARIA

\title{
L'ENNUI ET LA TÉLÉVISION D'ENJEU : UNE ÉTUDE À PARTIR DU GENRE SOAP OPERA
}

\section{Emmanuel Belin ${ }^{1}$}

L'analyse que nous présenterons ici ne consiste pas à relever les caractéristiques formelles d'un genre qui a déjà fait l'objet de nombreux commentaires. Plutôt, nous nous servirons des soap operas comme d'une métaphore pour comprendre l'une des significations possibles que peut prendre l'expérience télévisuelle dans la vie quotidienne. L'hypothèse principale sera que les textes de ce genre tirent leur attrait de leur capacité à créer des univers où "des choses importantes se passent" -nous utilisons dans ce sens l'expression : "télévision d'enjeu", télévision qui "met de l'enjeu" dans une existence habitée par l'ennui. Après une brève explication relative à la notion de genre, nous passerons en revue les caractéristiques essentielles des soap operas, telles qu'elles ont été mises en évidence dans l'abondante littérature qui s'y rapporte, pour montrer qu'elles renvoient toutes à cette idée de "fabrication d'univers". Enfin, dans la troisième partie de ce texte, nous nous attacherons à préciser ce qu'il faut entendre par "créer de l'enjeu", à partir d'une théorie de l'ennui et du jeu winnicottien.

\footnotetext{
1 Aspirant du Fonds National de la Recherche Scientifique.
} 


\section{Brève mise au point sur la notion de genre}

L'expérience télévisuelle est pour nous un fait social; elle peut être caractérisée plus précisément comme l'expérience faite de genres télévisuels. Cela implique évidemment d'élargir considérablement la portée de la notion de genre, de l'extraire du giron de l'analyse textuelle pour mettre en évidence sa nature sociologique. Sept élargissements nous paraissent indispensables pour sortir de l'aporie bien connue de la classification générique, selon laquelle un genre "se définit en termes de traits communs à un ensemble de textes identifiés par l'analyste comme contenant les traits définissant le genre (n.t.)"'. Ces élargissements sont : 1) l'approche ethnométhodologique qui fait du genre une activité sociale de classification par laquelle les acteurs sociaux ordonnent leur expérience du monde ${ }^{2}$-et à ce titre s'engagent dans un champ de rapports de forces symboliques au travers de stratégies de légitimation ${ }^{3}$;2) l'approche en termes de conventions qui fait du genre un des pivots organisateurs de la relation entre la production et la consommation de produits culturels en rendant possible leur programmation ${ }^{4}$; 3) l'approche en termes de prototypie, développée par les sciences cognitives, selon laquelle les catégories ne se définissent pas par le croisement de traits définitoires abstraits, mais plutôt s'organisent autour de noyaux représentationnels qui sont les «meilleurs représentants» de la catégorie, dont ils constituent les traits saillants, et par rapport auxquels des formes moins centrales sont

1 R. C. AlLEN, "Bursting Bubbles : «Soap Opera», Audiences, and the Limits of Genre", in E. SeITER, H. Borchers, G. Kreutzaner, E.-M. WARTH (eds.) Remote Control : Television, Audiences, and Cultural Power, London, Routledge, 1990, p. 47 ; voir aussi : A. Tudor, "Genre and Critical Methodology" in B. NiCHOLS (ed.) Movies and Methods, Berkeley, University of California Press, 1976, p. 118126.

2 On se réfère ici à la tradition ethnnométhodologique telle que présentée par $\mathrm{H}$. GARFINKEL, Studies in Ethnomethodology, Cambridge, Polity Press, 1984 (1967); A. Schurz, Le chercheur et le quotidien. Phénoménologie des sciences sociales, Paris, Méridiens Klincksieck, 1987 ; P. BERGER \& Th. LUCKMANN, La construction sociale de la réalité, Paris, Méridiens Klincksieck, 1986.

3 On reconnaît l'appropriation par Bourdieu des thématiques de classification. Bourdieu est une source d'inspiration directe des travaux des cultural studies anglosaxonnes auxquels il sera fait abondamment référence dans la suite.

4 St. Neale, "Genre and Cinema" in T. BennetT et al. (eds.), Popular Television and Film, London, British Film Institute, p. 6-25 ; J. FISKE, Television Culture, London - New York, Routledge, 1987; sur la programmation: G. LOCHARD \& H. BOYER, Notre écran quotidien. Une radiographie du télévisuel, Paris, Dunod, 1995. 
ordonnancées par variations métonymiques ${ }^{1}$; 4) toujours au sein des sciences cognitives, l'étude pragmatique de l'expérience télévisuelle comme situation communicationnelle caractérisée par l'indécidabilité sémantique, le genre intervenant dans la constitution même du sens du texte comme métadiscours sur les limites de l'inférence qu'est la réception et sur les voies prioritaires qu'elle doit emprunter ${ }^{2} ; 5$ ) l'approche plus psychanalytique qui s'articule autour du concept de jeu, définit le genre comme condition du passage du play au game et, à ce titre, le pose comme un des éléments essentiel du plaisir de la lecture, faisant du texte un objet transitionnel complexe, une «illusion» (in-ludio) à laquelle on est susceptible de se prendre et qui permet l'assomption du paradoxe entre le cognitif ("je sais bien...") et l'affectif ("mais quand même...") ; 6) l'approche ethnographique qui définit le genre comme l'ensemble des moteurs de socialisation qui rassemblent des publics et constituent leur idioculture, leurs normes et leurs références obligées $\left.{ }^{4} ; 7\right)$ enfin, un dernier amendement à la notion de genre se rapporte au "mode de ligation" des textes qui le définissent : il s'y agit d'insister sur le fait que ce qui rassemble les textes dans un genre est d'abord une logique affective et ensuite seulement une logique cognitive -le genre devenant ainsi un système de connotations qui permet de n'aller que vers ce qu'on aime et d'éviter ce qu'on n'aime pas.

Ce qui doit apparaître dans cette énumération un peu sybilline ${ }^{5}$, c'est l'opportunité d'une étude des genres télévisuels comme formes

1 G. LAKOF, Women, Fire, and Dangerous Things. What Categories Reveal about the Mind, Chicago - London, The Univesity of Chicago Press, 1987 ; en français, une présentation informée, quoique orientée surtout vers la linguistique, peut être trouvée chez: G. KLEIBER, La sémantique du prototype. Catégories et sens lexical, Paris, P.U.F., 1990.

2 D. SPERBER \& D. WILson, Relevance. Communication and Cognition, Oxford, Basil Blackwell, 1986.

3 On se réfère ici à : M. PICARD, "Lector ludens", Autrement, n' ${ }^{\circ} 153$ (1993), p. 135136 ; M. PICARD, "La lecture comme jeu”, Poétique, n 58 (1984), p. 260 ; sur la notion d'objet transitionnel : D. W. WinNicotT, Jeu et réalité, Paris, Gallimard, 1975 ; sur la notion d'illusion : J. HuIzINGA, Homo Ludens, Paris, Gallimard (Tel), 1951 ; sur le texte comme espace potentiel : O. MANNONI, Clefs pour l'imaginaire ou l'Autre Scène, Paris, Éd. du Seuil, 1969, p. 9-33.

4 Sur la notion d'idioculture : G. A. FINE \& S. KLEINMAN, "Rethinking Subculture: An Interactionist Analysis", American Journal of Sociology, n'85 (1979), p. 1-20; sur l'approche ethnologique : voir infra, C. PENLEY, D. D. BIELBY, H. JENKINS.

5 Nous avons développé cette approche large du genre dans: E. BELIN, Une sociologie des espaces potentiels. Télévision dispositive et expérience ordinaire, Dissertation doctorale, U.C.L., 1997, p. 328-353. 
significatives de la socialité contemporaine. Notre hypothèse générale est que l'expérience télévisuelle, telle qu'elle se structure par les genres, est un dispositif mis en place par la société moderne avancée pour jouer le rôle de relais du monde de la vie, de foncteur de continuité de l'expérience. Ici encore, il nous faut être très succinct ; disons simplement que sans de tels dispositifs l'existence serait vertigineuse. D'autres relais existent dans la société moderne -la psychothérapie, l'intelligence artificielle, le jeu vidéo, le sport, les psychotropes...comme d'ailleurs, avec d'autres ressources, dans les contextes traditionnels -la prière collective ou individuelle, l'animal domestique ou le totem, la danse, le travail, les drogues naturelles ${ }^{1}$... Toutes ces formes culturelles peuvent être comprises comme des espaces potentiels où se mettent entre parenthèses les évidences pratiques qui gouvernent notre rapport au monde et où se rétablit l'illusion d'un univers néguentropique, bienveillant à notre égard ${ }^{2}$. Il semble bien que la modernité puisse être comprise comme une gigantesque entreprise de dissolution de ces aires intermédiaires, confinées à une expérience esthétique de plus en plus dissociée de tout ancrage éthique ou cognitif, et à ce titre, de plus en plus formaliste ${ }^{3}$. Suivant

1 Citons parmi d'autres travaux, à propos des psychothérapies: A. GidDENS, Modernity and Self-Identity, Stanford Ca, Stanford University Press, 1991 ; J.-M. LACROSSE, "Les psychothérapies et l'anthropologie de la subjectivite", in E. BELIN \& G. BAJOIT (eds) Contribution à une sociologie du sujet, Paris, L'Harmattan, 1997, p. 279-296; à propos de l'intelligence artificielle: Sh. TuRKLE, The Second Self. Computers and the Human Spirit, New York, Simon and Schutster, 1984 ; C. PENLEY \& A. Ross, "Cyborgs at Large: Interview with Donna Harraway", in C. PENLEY \& A. Ross (eds.) Technoculture, Minneapolis - Oxford, University of Minnesota Press, 1991, p. 1-20; L. SFEZ, Critique de la communication, Paris, Éd. du Seuil, 1988; à propos des jeux vidéo: Sh. TuRKLe, op. cit.; M. KINDER, Playing with Power in Movies, Television and Video Games, Berkeley - Los Angeles - Oxford, University of California Press, 1991; à propos des psychotropes : A. EHRENBERG, L'individu incertain, Paris, Calmann-Lévy, 1995 ; à propos de la prière, nous avons écrit deux textes : E. BELIN, "Faire parler l'absence", Recherches en communication, $\mathrm{n}^{\circ} 2$ (1994), p. 171-188; E. BELIN, "Conservatisme et chemises à fleurs : figures de la pratique religieuse à quelques miles de Silicon Valley", Revue nouvelle, n¹2 (1996), p. 74-84; pour une comparaison entre totems et objets techniques, on lira: Br. LATOUR, Petite réflexion sur le culte moderne des dieux faitiches, Ed. Synthélabo (coll. Les empêcheurs de penser en rond), 1996.

2 Nous développons la thématique de la bienveillance dans : E. BELIN, "L'expérience dispositive", in G. BAJOIT (ed), Actes du colloque "La socialisation des jeunes dans un monde en mutation", Éditions Luc Pire, 1997 (à paraître).

3 Nous nous appuyons pour dire cela sur la synthèse des théories de la modernisation entreprise par J. HABERMAS, Théorie de l'agir communicationnel, Tome I : Rationalité de l'agir et rationalisation de la société, Éd. Fayard, Paris, 1987 (tr. fr.). 
l'hypothèse de B. Latour, nous arguerons qu'à ce processus de purification s'est adjoint, en sous-main, un processus de prolifération de formes hybrides où se réunissent l'humain et le non-humain' ${ }^{1}$. Pour nous, ces formes hybrides se sont positionnées dans la vie sociale comme des remparts contre deux formes de souffrance propres à la modernité "désenchantée": l'ennui et l'anxiété2. Les genres télévisuels, on l'aura compris, nous semblent participer de ce mouvement. Pour R. Kubey et M. Csikszentmihalyi ${ }^{3}$, les formes télévisuelles en sont des modalités faibles mais constantes, les "flow experiences" intenses étant réservées à des situations plus interactives comme les jeux vidéo. Il nous semble qu'en élargissant comme nous l'avons fait la notion de genre, nous pouvons mettre en évidence un continuum d'expériences qui va de la détente à l'excitation, et qui toujours présente des alternatives aux formes de vertige que sont l'ennui et l'anxiété.

Certains genres télévisuels s'orientent, nous semble-t-il, plutôt vers les problématiques de l'anxiété liée à l'incertitude, à la disparition des repères normatifs, à l'inflation des exigences portées sur l'individu que provoque la dualisation et la désinstitutionnalisation de la société ${ }^{4}$. Nous parlons pour désigner ces genres de télévision

Le symptôme de cette "bonne foi" sous-jacente à la rationalisation nous semble être la nécessité qu'éprouve Habermas de chapeauter les rationalités cognitive, éthique et esthétique par la rationalité cognitive -toute prétention à la validité étant lue en termes d'assertion de vérité : il est vrai que cela est beau, bien, vrai. Dans les formes transitionnelles, la forme canonique serait plutôt : il est beau que cela soit vrai, bien, beau; l'usage du subjonctif dans la seconde proposition traduit la difficulté qu'on éprouve à manifester symboliquement la posture mentale prélangagière qui est prise dans ces formes de jugement. Autrement dit, la nature même de la reconstruction habermassienne le force à ses conclusions.

$1 \mathrm{Br}$

Br. LATOUR, Nous n'avons jamais été modernes, Paris, La Découverte, 1995.

2 Cette conceptualisation s'inspire de : M. CsIKszentmiHALYI, Beyond Boredom and Anxiety, The experience of Play in Work and Games, San Francisco - Washington London, Jossey-Bass Publishers, 1975.

3 R. KubEY \& M. CSIKSZENTMIHALYI, Television and the Quality of Life. How Viewing Shapes Everyday Experience, Hillsdale - New Jersey, Lawrence Erlbaum Associates, 1990.

4 On se réfère évidemment aux travaux de A. EHRENBERG, L'individu incertain, op. cit.; A. EHRENBERG, Le culte de la performance, Paris, Calmann-Lévy, 1991 ; P. CHAMBAT \& A. EHRENBERG, "Les reality shows, nouvel âge télévisuel ?", Esprit, $\mathrm{n}^{\circ} 1$ (1993), p. 5-12 (numéro spécial). On peut aussi citer D. CARDON, "«Chère Ménie...» Émotions et engagements de l'auditeur de Ménie Grégoire", Réseaux, $\mathrm{n}^{\circ} 70$ (1995), p. 41-77; D. MeHL, La télévision de l'intimité, Paris, Éd. du Seuil, 1996 ; R. Silverstone, Television and everyday life, New York - London, Routledge, 1994. 
d'emprise ou de télévision prothétique, dans le sens où l'expérience télévisuelle y apparaît comme une manière d'accroître l'emprise des individus sur leur existence et de s'orienter dans leur vie $1^{1}$. On pense, par exemple, aux reality shows, aux informations ou à certains sitcoms ${ }^{2}$; ce n'est pas de ces formes qu'on parlera ici. Nous consacrerons cette réflexion à ce que nous appelons la télévision d'enjeu, qui se construit en contrepoint d'une problématique de l'ennui ordinaire. Le soap opera nous paraît être un bon exemple de ce type de topique, même si des aspects d'emprise peuvent s'y découvrir et, parfois même, y jouer un rôle important ${ }^{3}$. Phénomène de société dans les pays anglo-saxons aussi bien que dans la culture hispanophone et lusophone, les soaps n'ont pas ce statut dans les pays francophones -exception faite, peut-être, du Québec. C'est donc à titre de métaphore que nous les étudierons ici : à maints égards, nous le verrons, ce genre peut être compris comme une forme particulièrement typique de l'expérience télévisuelle, une sorte d'idéal-type. Par télévision d'enjeu, nous entendons que la télévision peut s'inscrire dans la durée de la vie quotidienne, s'y instiller comme une expérience à part entière dont le statut de réalité n'est pas moindre que celui d'autres expériences comme, par exemple, les conversations entre amies ou la vie conjugale. Nous utilisons le terme enjeu pour signifier que, face à l'ennui, c'est-à-dire à l'impossibilité d'utiliser à plein rendement une série de compétences sociales par défaut de

1 Pour un développement de cette dichotomie, on pourra se référer à : E. BELIN \& L. VAN CAMPENHOUDT, "La télévision entre espace public et vie quotidienne", in $\mathrm{H}$. DUMONT \& A. STROWEL (eds) Politique culturelle et droit de la radio-télévision, Bruxelles, Publications des Facultés Universitaires Saint-Louis, 1997 (à paraître).

2 On pense aux travaux de D. PASQuiER, "Hélène et les garçons : une éducation sentimentale", Esprit, n6 (1994), p. 125-144 ; D. PASQUIER, "«Chère Hélène» Les usages sociaux des séries collège", Réseaux, nº 70 (1995), p. 9-39; Fl. DUPONT, "Hélène et les garçons: une fiction initiatique ?", Socio, déc. 1994, p. 3-11. Par certains de leurs aspects, ces fictions relèvent de la télévision d'enjeu-songeons, par exemple, à l'abondance des textes secondaires, à la confusion entre personnages et acteurs, ou encore aux télescopages diégétiques entre séries. Mais les aspects que relèvent ces auteurs participent pour l'essentiel de l'emprisecomme le révèle l'emploi de mots comme "usages sociaux", "fiction initiatique" ou encore "éducation sentimentale".

3 Ainsi, pour Giddens, "il va de soi que les soap operas (...) sont des fuites - des substituts à des satisfactions réelles qui ne peuvent être obtenues dans des conditions sociales normales. Cependant, le plus important réside dans les formes narratives typiques qu'ils offrent, et qui suggèrent des modèles pour la construction de récits du self'- il s'agit bien ici d'emprise (A. GidDENs, Modernity and SelfIdentity, Stanford, Stanford University Press, 1991, p. 199. 
sollicitations de l'environnement, de telles formes offrent à leur public de nouvelles ressources symboliques qui lui permettent de vivre des expériences sur le mode de l'importance et de l'implication plutôt que sur celui du blasement ou de l'indifférence, modes qui relèvent de l'angoisse et du vertige. Ainsi donc, ce que nous appelons "télévision d'enjeu" ou "télévision dispositive" dépasse le seul genre "soap", et peut nous servir de grille de lecture de toute une série d'autres formules de l'expérience télévisuelle.

\section{La télévision dispositive ou d'enjeu}

Sans prétendre clôturer un débat particulièrement intense, nous allons dans un premier temps mettre en avant quelques acquis des recherches empiriques qui ont été entreprises sur les soap operas, tant au niveau de leur structure textuelle qu'à celui de leur réception -on le verra ainsi concrètement : dans la pratique du terrain, les chercheurs sont amenés à utiliser spontanément une définition élargie du genre. Quatre questions nous semblent couvrir l'essentiel des réflexions et des études entreprises: celle de la clôture du texte, celle des transgressions et des brouillages entre les univers textuel et historique, celle de la structuration en "acteur social" des communautés de réception spécifiques de ce genre, et enfin celle du contexte existentiel dans lequel s'inscrit cette réception. Chaque fois, nous chercherons, au-delà du simple relevé bibliographique, d'indiquer en quoi ces questions "classiques" renvoient à notre hypothèse de la télévision d'enjeu. À partir de là, nous pourrons ensuite, dans la troisième partie de cet article, développer notre approche de la place qu'occupe l'expérience télévisuelle dans le cours de l'existence ordinaire.

\subsection{La question de la clôture}

Le caractère "ouvert" des soaps est une des caractéristiques les plus souvent citées du genre. Cela ne signifie pas que ces séries ne finissent jamais, mais plutôt qu'elles ne sont pas faites pour finir ${ }^{1}$.

1 Cf. D. PORTER, "Soap Time: Thoughts on a Commodity Art Form", College English, 38 (8), 1977, p. 783 ; J. BuTLER, "Notes on the Soap Opera Appartus : Televisual Style and «As the World Turns»", Cinema Journal, 25 (3), 1986, p. 54. Ceci ne s'applique pas aux télénouvelles latino-américaines, qui sont généralement organisées en vue d'une "fin" lointaine -au bout de quelques centaines d'épisodes. 
Contrairement aux récits classiques, elles n'évoluent pas vers une clôture narrative, et peuvent se poursuivre plusieurs dizaines d'années ${ }^{1}$. La longueur de la série est à comprendre comme un indice d'une particularité structurelle que Allen appelle la "complexité paradigmatique", dont il note diverses implications : a) il n'y a pas de personnage central, mais une communauté parfois nombreuse de personnages dont les rapports interpersonnels se déploient au fil des ans en une combinatoire infinie ; b) tout événement est susceptible d'être réinterprété, voire nié ${ }^{2}$-les personnages morts pouvant même, au besoin, être ressuscités ; c) chaque nouvel événement ne peut être pleinement compris qu'en référence à la "mémoire historique" de la série tout entière ${ }^{3}$; d) l'aspect communautaire induit par l'ouverture narrative conduit le plus souvent à une focalisation du récit sur des lieux plus que sur des personnages -le restaurant, l'hôpital, etc. ; e) l'absence de "fin" réduit fortement les possibilités de tirer des "morales", des "messages" précis et définitifs, même si de fortes connotations morales sont sans cesse perceptibles ${ }^{4}$. De nombreuses publications traitent en détail cette question, aussi nous ne nous y attarderons pas.

Nous le verrons plus loin, cette absence de clôture a de nombreuses conséquences sur les autres aspects caractéristiques du genre ; pour le moment, contentons-nous de noter quelques implications de cette question sur le type d'expérience faite de la télévision à travers les soaps. Notre hypothèse sera ici que le caractère

1 Cf. General Hospital, le détenteur du "record" en la matière : produit par ABC depuis 1963 , il est encore aujourd'hui diffusé une heure par jour, cinq jours par semaine!

2 D. C. ReEp, "The Siren Call of the Super Couple : Soap Operas' Destructive Slide Toward Closure", in S. FRENTZ (ed.), Staying Tuned : Contemporary Soap Opera Criticism, Bowling Green, Bowling Green State University Popular Press, 1992, p. $96-102$.

3 R. C. Allen, "Introduction", in R. C. Allen (ed.), To be continued... op. cit.

4 Pour Laura Stempel-Mumford, l'idée de clôture existe dans les soap operas, mais se réfère à des storylines précises. Le plus souvent, une douzaine de storylines sont explorées alternativement dans un soap, au rythme de deux à trois storylines par épisode. Si certaines d'entre-elles ne se "fermaient" pas, argumente cet auteur, la série dans son ensemble ne pourrait que s'enliser. Certes, toute histoire fermée peut être, au besoin, ré-ouverte ultérieurement. Cependant, le "geste" de la clôture est omniprésent et joue un rôle essentiel dans le déroulement paradigmatique de l'histoire. À partir de là, Stempel-Mumford établit une typologie des "clôtures" de storylines, dont aucune n'est définitive. (Laura Stempel-Mumford, "How Things End : The Problem of Closure on Daytime Soap Operas", Quarterly Review of Film \& Video, vol 15, $\mathrm{n}^{\circ} 2$ (1994), p. 57-74). 
"combinatoire" du monde fictionnel est le reflet du caractère également combinatoire de la vie quotidienne et, plus largement, du monde vécu. Ainsi, pour Jesús González Requena, "les personnages du culebrón ${ }^{1}$ existent, coexistent avec le spectateur au sein même de son temps quotidien : chaque nouvel épisode est, dès lors, une rencontre rassurante avec ces êtres électroniques qui se sont introduits dans notre quotidienneté (...). L'univers narratif du culebrón, à se reproduire sans cesse, tend à se constituer en un univers autosuffisant qui se rapporte à lui même, abolissant finalement la réalité et se constituant en référent unique de lui-même (n.t.)"2. Se référant à la psychanalyse lacanienne, Requena voit dans ce processus une dérive incestueuse et une régression imaginaire ${ }^{3}$. Sans nier la force et l'intérêt de sa démonstration, la chose nous semble ne pas pouvoir être réduite à cette interprétation tragique. Le caractère incestueux de ces séries, qu'il interprète très largement comme l'abolition des interdits permettant l'entrée dans le symbolique ou, pour parler simple, l'instauration du "tout est permis", est renvoyé à ce qu'on admet généralement être la logique schizoïde du capitalisme : l'engloutissement de tout le possible, l'asservissement des choses et de leur contraire, le remplacement du sujet divisé par le sujet éclaté, ce qui revient à l'abolition du sujet ${ }^{4}$. Or, nous semble-t-il, le marché n'est pas la seule formule sociale intrinsèquement schizoïde ; le sens commun, le monde vécu, la quotidienneté qu'on y oppose traditionnellement, partagent cette caractéristique. Ainsi, remarque François Lyotard, la sagesse populaire n'affirme-t-elle pas en même temps que "tel père, tel fils" et qu' "à père avare, fils prodigue" 5 ? On pourrait chercher loin l'origine

1 Littéralement : "couleuvre". Ce terme est utilisé en espagnol pour désigner un récit qui prétend ne jamais s'achever, qui "s'enroule sur le désir du spectateur et, une fois qu'il l'a attrapé, se reproduit indéfiniment en s'enroulant sur lui-même, sur ses personnages, sur ses anecdotes narratives" (n. t.), (J. GonZÁlEZ REQUENA, El discurso televisivo: espectáculo de la posmodernidad, Madrid, Cátedra, 1995, p. 120).

2 Ibid., p. 122-123.

3 Cette thématique de l'inceste est récurrente. Ainsi, Sabine Chalvon-Demersay estelle parvenue à un constat du même genre (l'abondance des relations de type incestueux) en analysant mille scénarios de feuilletons télévisés récoltés dans le cadre d'un concours (S. Chalvon-Demersay, Mille scénarios, Paris, Métailié, 1995). Voir également la lecture de M. Augé, La traversée du Luxembourg, Paris, Hachette, 1985, p. 156-157.

4 Cette thématique est reprise de G. Deleuze \& F. GuatTari, Capitalisme et Schizophrénie. Mille Plateaux, Paris, Éd. de Minuit, 1980. 5 J.-Fr. LyOTARD, Le Postmoderne expliqué aux enfants, Paris, Galilée, 1986, p. 40 et
passim. 
de cette négation du principe de non-contradiction dans le monde vécu ; nous nous contenterons ici de souligner que cette négation est centrale : elle montre que le monde vécu ne s'oriente pas tant vers la connaissance du monde que vers l'établissement de modus vivendi à son égard, ou pour le dire plus précisément, l'entretient d'un "goût de vivre" qui a réponse à tout -une foi qui ne se prive pas du recours à la mauvaise foi en toute bonne foi.

Ce que les anglo-saxons appellent l'open-endedness de ces textes nous semble plus qu'un signe distinctif par rapport à d'autres formes narratives. $M$. Kinder parle de genres narratifs intrinsèquement combinatoires -elle rejoint ainsi Requena ; elle la caractérise comme un sleep bargaining genre, ce genre de récits "à rallonges" que l'enfant raconte pour retarder le moment où ses parents s'éloigneront de son lit pour le laisser dormir. "Dans toutes ces fictions de «négociation du sommeil», c'est le langage et sa capacité de générer un nombre indéfini de combinaisons et un flux infini de récits qui maintient la conscience et le contrôle du locuteur, et diffère le

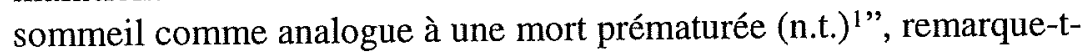
elle à partir d'une métaphore des Mille et une nuits. Kinder développe son argumentation à propos des jeux vidéo et des émissions pour enfants, et c'est de fait à ce type de programmes qu'elle s'applique le mieux. Il ne nous semble toutefois pas impossible d'élargir le champ aux daytime soaps, et aux "couleuvres" télévisuelles dans leur ensemble, qui relèvent d'une promesse sans cesse réitérée dont la réalisation est sans cesse différée ; le personnage de Schéhérazade est ici aussi central, puisqu'elle conte pour ne pas mourir, exactement comme l'enfant dans son lit. L'open-endedness du texte télévisuel le rattache au monde de la vie comme la garantie d'une abondance de significations possibles, comme promesse qu'il se passera toujours quelque chose, ou pour prendre une métaphore thermodynamique, que l'action homogénéisante de l'entropie ne viendra jamais à bout de la tendance à la différenciation et à la mise en tension de différences de rapports qui caractérise la vie. Nous verrons plus loin l'importance de cette idée d'abondance, de garantie, dans une théorie de l'ennui.

1 M. KInder, Playing with Power in Movies, Television, and Video Games, Berkeley - Los Angeles - Oxford, University of California Press, 1991, p. 20. 


\subsection{La question de la transgression et du brouillage}

"La jolie Ava devrait être ressuscitée", lisait-on au hasard, ce printemps dernier, dans le courrier des lecteurs de Soap Opera Magazin, avec sous la photographie de la jolie Ava : Je suis une fan fidèle de The City, et je ne comprends pas qu'ils ne reprennent pas Ava. Je ne saurais pas dire tout le bien que je pense de la belle, talentueuse Lisa Peluso. J'aimerais la voir en couple avec le tout aussi sexy et talentueux Roscoe Born (...). La storyline avec Alex et Jocelyn est le plus gros flop de tous les temps. Ramenez Ava et mettez-là en couple avec Nick, et vous verrez les cotes d'audience monter en flèche! (n.t.)"'. L'absence de marqueurs textuels permettant de savoir si ce dont on parle relève de la réalité ou de la fiction est caractéristique de l'écriture des fanzines en tous genres. Dans le cas précis des soaps, ce qui frappe, c'est la cohérence, la permanence de la coexistence de deux ordres textuels -un premier compris surtout dans les textes primaires, et le second relevant essentiellement des textes secondaires- qui s'appuient l'un sur l'autre pour donner d'étranges discours tels que celui que nous venons de mentionner. La raison en est simple : une des caractéristiques de la production des soaps est que le "tournage", et même l'élaboration du scénario, sont plus ou moins co-occurrents à l'évolution du récit. On se retrouve avec quelque chose qui n'est pas loin de l'idée de "troupe", mais une troupe qui passe une bonne partie de la journée sous le feu des caméras, jouant des scènes où la personnalité des acteurs est moins "encadrée" que dans une fiction classique, du fait des conditions de production. Pour les fans, le texte diffusé est ainsi l'occasion de construire un second texte, l' "histoire du tournage", qui est réelle celle-là, et fait l'objet de stratégies narratives. Or, il semble bien que court-circuiter ces deux séries soit une source non négligeable de plaisir des fans, et non de confusion. Ainsi, C. Harrington et D. Bielby montrent l'attrait particulier des spectateurs pour les romances entre acteurs; les grossesses d'actrices sont des moments forts, parce qu'ils impliquent l'adaptation de scénarios et des jeux sur les personnages; les recastings pour raisons diverses sont l'objets de polémiques passionnées. Pour Bielby, la transgression incessante de la frontière entre réalité et fiction

1 “The Soap Box — «Lovely Ana Should be Reborn»", Soap Opera Magazine, Apr. 30, 1996, p. 22. 
n'aboutit pas à son effacement, mais bien à son renforcement ${ }^{1}$. Une autre sorte de téléscopage nous paraît relever cette fois du brouillage, il s'agit de ce que L. Soarès appelle l' "enchâssement"2, l'utilisation comme ressort narratif d'éléments identifiables de la réalité quotidienne du récepteur. Il s'agit pour elle surtout de publicité, mais l'enchâssement dépasse de loin cette intention et doit être compris comme une des figures de style caractéristique de certains genres télévisuels contemporains ${ }^{3}$. C'est d'ailleurs surtout à cet enchâssement non publicitaire que recourent les autres genres proches comme le soap ou certains sitcoms, ce qui démontre qu'on a bien affaire à une forme narrative spécifique» ${ }^{4}$.

Si nous reprenons la distinction entre textes primaires et textes secondaires que propose Fiske, la transgression consiste à maintenir une ambiguité dans l'intertextualité verticale, entre le "texte" et le "texte du texte" -par exemple : l'"aventure» du tournage de la série, telle qu'elle est relatée par les fanzines, ou encore la préparation des vedettes pour les soap opera awards tenus une fois l'an. Dans le brouillage, c'est également d'ambiguité qu'il est question, mais celleci s'opère dans l'intertextualité horizontale, entre le "texte" et le "contexte", ce que Fiske appelle le texte tertiaire. L'idée d'"enchâssement" de Soarès doit ainsi se comprendre comme un brouillage et non comme une transgression, car elle consiste à incorporer au monde diégétique des éléments du contexte historique de telle manière que ceux-ci acquièrent eux-même une connotation positive ${ }^{6}$. Dans un cas

1 C. L. Harrington \& D. D. Bielby, Soap fans. Pursuing Pleasure and Making Meaning in Everyday Life, Philadelphia, Temple University Press, 1995, p. 48-59.

${ }^{2}$ L. SOARÈS DE SOUSA, Représentation et idéologie. Les téléromans au service de la publicité, Montréal, Les Éditions Balzac, 1994, p. 213.

3 Cf. A. Guillermoprieto, "Obsessed in Rio", The New Yorker, Aug. 16, 1993, p. 44-55. On y montre entre autres comment le président Collor devient malgré lui star d'une telenovela.

4 L'actualité est souvent, d'une manière ou d'une autre, intégrée aux événements diégétiques des soaps. Cf. L. SPENCE, “«They Killed off Marlena, But She's On Another Show Now». Fantasy, Reality, and Pleasure in Watching Daytime Soap Operas", in R. C. AlLEN (ed.), To be continued..., op. cit., p. 182-198.

5 "L'intertextualité verticale consiste dans les relations du texte primaire avec d'autres textes qui s'y réfèrent spécifiquement. Ces textes secondaires, comme la critique ou la publicité, travaillent à promouvoir la circulation de significations particulières du texte primaire. Les textes tertiaires sont le niveau final, crucial de cette circulation, dans le sens où ils ont lieu au niveau du spectateur et de ses relations sociales (n.t.)" J. FiSKE, op. cit., p. 117.

6 Ainsi, dans une télénouvelle, on reconnaît la marque des vêtements des gentils, mais pas celle de ceux des méchants. Nous avons par ailleurs nuancé ce propos en 
comme dans l'autre, ce qu'il faut souligner, c'est que le plaisir et la constitution du sens ne résultent pas seulement du processus mimétique propre à toute narration, et que développe P. Ricœur dans sa théorie des trois mimèsis, mais également d'un jeu continuel sur le double statut du texte, entité qui vaut tant comme signifiant que comme signifié ${ }^{1}$. Cela renvoie évidemment à la notion d'espace potentiel telle que la propose Winnicott -et peut-être plus adéquatement en l'occurrence, à celle de jeu dont nous avons fait une des dimensions du genre. Nous pourrions sans doute, pour reprendre les catégories de Picard, formuler l'hypothèse que le brouillage se rapporte au play tandis que la transgression est relative au game, mais démontrer cela nous emmènerait trop loin. Nous proposons d'emprunter le raccourci que nous proposent $V$. Nguyên-Duy et S. Cotte : ces deux chercheuses ont observé avec étonnement un retour des marqueurs fictionnels dans les textes secondaires relatifs à un téléroman québécois, Scoop, ces dernières années; elles ont en outre constaté un effacement de ces marqueurs dans les textes secondaires relatifs à une série humoristique, La petite vie. La raison, expliquentelles, est simplement la chute de popularité de Scoop, tandis que $L a$ petite vie grimpait dans les sondages ${ }^{2}$. Cette anecdote indique que le jeu aux frontières n'est possible que dans un univers d'évidence, un univers où les référents sont suffisamment partagés pour que l'indexicalité soit résorbée pragmatiquement. Il faut qu'existe une "connivence" pour que le jeu prenne.

\subsection{La question de l'interactivité et des communautés d'interprétation}

Dans le champ des soaps, la question de l'interactivité nous semble devoir être posée de pair avec celle des communautés d'interprétation. Certes, des formes de feed-back du public existent,

insistant sur les logiques de réception qui peuvent conduire à adopter des comportements promus dans les télénouvelles non par "aliénation" mais par simple calcul rationnel d'anticipation de la norme (E. BELIN, "Norme et médias", in J. DE MUNCK \& M. VERHOEVEN (eds.), Les mutations du rapport à la norme, ParisBruxelles, De Boeck \& Larcier, 1997, p. 87-102.

1 Nous ne pouvons pas développer ici la notion d'identité narrative de P. RICÆEUR, Temps et Récit, Paris, Éd. du Seuil, coll. "Points", 3t., 1983.

2 V. NGUYÊn-DuY \& S. COTTE, "Le discours de presse sur les téléromans : le cas de la télésérie Scoop”, Communication, vol. 16 (2), 1996, p. 189-209. 
comme l'audimétrie, les choix de scénarios dans certaines séries expérimentales, ou bientôt le multiplexing que rend possible la multiplication des canaux disponibles. Nous pourrions mettre en avant, à partir de différentes recherches, notamment dans le domaine des télécommunications et de la réalité virtuelle, que cet accroissement de l'interactivité peut se comprendre à maints égards comme l'établissement des conditions de possibilité de l'expérience phénoménologique d'un monde. Cependant, l'essentiel, pour le moment, ne nous paraît être là. 'L'interactivité réelle nous importe peu ; ce qui nous intéresse ici, c'est le sentiment de participation à l'histoire que peuvent éprouver les spectatrices, c'est-à-dire ce que les anglo-saxons appellent l'agency, le fait d'être acteur. Nous définirons grossièrement l'agence comme la superposition momentanée ou durable de la quotidienneté et de l'historicité1. Comme le montre bien H. Jenkins, le "fan" a toujours été représenté comme la figure même de l'aliéné, de celui qui ne parvient pas même à "vivre une vie" et doit, par conséquent, vivre celle qu'on lui fait ${ }^{2}$. Au contraire, montre-t-il par diverses recherches empiriques, les fans développent individuellement, mais surtout collectivement, des formes d'agence qui sont de l'ordre de la tactique au sens de M. de Certeau. L'ouvrage de Jenkins nous semble devoir être considéré comme séminal ; deux grandes lignées s'y enracinent, qui se distinguent par leur définition de l'action dans la réalité. La première lignée est sans doute la plus proche de l'intention de Jenkins; nous y retrouvons des auteurs comme C. Penley ainsi que maints tenants des cultural studies américaines. Penley étudie ce que produisent certaines fans féminines de la série de science-fiction Startrek, montrant ce que ces textes contiennent d'affirmation d'identité féminine -en l'occurrence, il s'agit de bandes dessinées, de romans, d'œuvres picturales érotiques mettant en scène une relation homosexuelle entre Kirk et Spock, les deux personnages centraux de la série ${ }^{3}$. Elle part d'une thématique du plaisir pour déboucher sur une thématique de l'action; en cela, son approche dépasse de loin les

1 Nous nous inspirons ici de la description a contrario du révolutionnaire entreprise par D. Martuccelli, Décalages, Paris, P.U.F., 1995. Nous aurions pu également prendre la description au premier degré du style Socialisme ou Barbarie, dans les années 70.

2 H. JenkIns, Textual Poachers. Television Fans and Participatory Culture, New York - London, Routledge, 1992 ; et notamment le premier chapitre : "«Get a Life !» : Fans, Poachers, Nomads", p. 9-49.

3 Une autre entrée par les textes secondaires est celle de C. BACON-SMITH, Enterprising Women, Philadelphia, University of Pennsylvania Press, 1992. 
lamentations de la pensée critique européenne en termes de dépolitisation et d'aliénation des masses. L'auteur se place dans une perspective féministe et cherche à "prendre ce fandom comme un cas exemplaire d'appropriation féminine de, de résistance à et de négociation avec la culture de masse" et estime que "le fandom, les diverses idéologies populaires de l'abus et du self-help, les philosophies New Age sont considérées comme bien plus pertinentes par rapports à leurs besoins et leurs désirs, que ce qu'elles perçoivent comme un féminisme de classes moyennes qui méprise la culture populaire et estime que la pornographie dégrade la femme ${ }^{1 "}$. Les communautés d'interprétation, que la littérature des cultural studies décrit depuis longtemps comme des lieux de constitution du sens, de négociation sémantique ${ }^{2}$, sont ici décrites comme des formes embryonnaires d'action politique ou, en langage tourainien, comme des lieux où se structurent une identité, une opposition et une totalité.

L'approche de Harrington et Bielby est sensiblement différente, et nous paraît s'inscrire mieux dans l'intuition de de Certeau, selon laquelle la production tactique se distingue de la stratégie par son absence de lieu, d'affirmativité, de constructivisme. Pour de Certeau, "[l] $\mathrm{a}$ «fabrication» à déceler est une production, une poiétique, -mais cachée, parce qu'elle se dissémine dans les régions définies et occupées par les systèmes de la "production» (télévisée, urbanistique, commerciale, etc.) et parce que l'extension de plus en plus totalitaire de ces systèmes ne laisse plus aux «consommateurs» une place où marquer ce qu'ils font des produits. À une production rationalisée, expansionniste autant que centralisée, bruyante et spectaculaire, correspond une autre production, qualifiée de "consommation": celle-ci est rusée, elle est dispersée, mais elle s'insinue partout, silencieuse et quasi invisible, puisqu'elle ne se signale pas avec des produits propres mais en manières d'employer les produits imposés

I C. Penley, "Feminism, Psychoanalysis, and the Study of Popular Culture", in L. Grossberg ; C. Nelson ; P.A. Treichler (eds.) Cultural Studies, New York London, Routledge, 1992, p. 492.

2 En ce qui concerne les fans, l'approche par les communautés d'interprétation est développée par T. LIEBES \& E. KATZ, The Export of Meaning. Cross-cultural Readings of DALLAS, New-York/Oxford, Oxford University Press, 1990. L'articulation entre communautés d'interprétation et la sociologie actionnaliste n'est guère opérée de manière explicite, si ce n'est, dans l'étude du genre «information», par W. A. Gamson, Talking Politics, Cambridge, Cambridge University Press, 1992. 
par un ordre économique dominant"'. Si le type de textes qu'étudient Jenkins ou Penley exige des femmes une lecture oppositionnelle ${ }^{2}$, les soaps, expliquent Harrington et Bielby, tant du fait de leur structure de textes destinés à un public féminin, que de par leurs caractéristiques de genre comme l'open-endedness, donnent lieu à relativement peu de productions personnelles de nature contre-culturelle. Cela ne signifie pas qu'aucune production discursive ne soit opérée, mais bien que le rapport entretenu à ces productions n'est pas celui qu'on entretient à une œuvre. Les productions sont comprises comme du bavardage (gossip), soit par téléphone ou autour d'une tasse de thé, soit dans les forums de discussion électroniques ${ }^{3}$, soit encore lors de rencontres dans les conventions organisées par les fan clubs, conventions qui font partie intégrante du processus de production et de diffusion du soap opera. Ainsi donc, c'est à une activité de résistance que se livrent les soap fans, une activité de reformulation et de participation plus ou moins fantasmatique à la production des textes primaires, plutôt qu'à un investissement dans la réalisation de textes secondaires. Faire pression sur les producteurs pour développer ou interrompre une storyline, établir des relations suivies avec une vedette ou un membre de l'équipe de tournage, écrire des lettres publiées dans le courrier des lecteurs des divers fanzines, mais surtout discuter avec d'autres fans des incohérences, des événements, de la psychologie des personnages, mettre en mots des fantasmes et des désirs,... voilà en quoi consiste l'action de la spectatrice. Ce que Bielby et Harrington essaient de faire comprendre, c'est que le soap fait partie de la vie quotidienne au même titre que les personnages "réels" de cette vie quotidienne que

1 M. DE CERTEAU, L'invention du quotidien, Paris, Union Générale d'Édition, 1985, p. 20.

2 Au sens de St. HALl, "Encoding, Decoding", in S. During (ed.) The Cultural Studies Reader, London - New York, Routledge, 1993, p. 90-103. Hall distingue une lecture dominante, qui se rapproche de la notion de décodage; une lecture négociée, qui opère un recadrage ; et une lecture oppositionnelle qui prend le texte à contrepied et construit le message comme le symétrique de l'intention perçue de l'émetteur.

3 D. D. Bielby \& C. L. Harrington, "Reach Out and Touch Someone : Viewers, Agency, and Audiences in the Televisual Experience", in J. CrUZ \& J. LEWIS (eds.) Viewing, Reading, Listening. Audiences and Cultural Reception, Boulder - San Francisco - Oxford, Westview Press, 1994 ; C. L. HARRingTon \& D. D. BielBY, "Where did you hear that? Technology and the Social Organization of Gossip", The Sociological Quarterly, 36/3, p. 607-628. 
sont le mari, les enfants, les amies ${ }^{1}$. L'émancipation, pour elles, s'opère au sein même du monde de la vie, et non dans ce qu'arbitrairement certaines classes sociales désignent comme l'histoire. Ces femmes sont décrites comme actrices parce qu'elles participent activement à la construction, l'orientation, l'entretien d'un monde où elles réalisent leurs rêves et confèrent une signification à leur existence. Elles ne sont pas aliénées parce qu'elles sont partie prenante dans la construction de l'illusion dans laquelle elles peuvent vivre, parce qu'elles parviennent à se maintenir dans cet espace flottant et vacillant de l'expérience diégétique d'un texte qu'elles contribuent elles-mêmes à produire, ou à tout le moins, dont elles connaissent les moindres détails des mécanismes de production.

\subsection{Les questions relatives au public}

Le caractère spécifiquement féminin des soaps a été maintes fois relevé : faible importance de l'action comparativement à la réaction et aux conséquences de l'action sur les différentes psychologies des personnages en réseau $x^{2}$, prévalence de la construction discursive verbale de la réalité et des événements, abondance des gros plans qui mettent en avant l'intimité et les sentiments des personnages ${ }^{3}$, fragmentation des récits qui correspond aux rythmes de la vie quotidienne de la femme au foyer, selon une conception non linéaire du temps, insistance sur des thématiques liées à la vie conjugale et domestique, etc. Pour T. Modleski, les soaps apportent à la mère la promesse de l'immortalité de la famille, et positionnent le lecteur dans la position de la mère idéale, qui comprend et encourage chaque membre d'une famille désunie et chaotique. Les intrigues développées dans les soaps concernent toujours la complexité et l'irréductibilité

1 On ne peut s'empêcher de penser aux conversations «de village». Là aussi, c'est à une véritable science généalogique, psychologique, sociologique et historique qu'il est sans cesse fait appel pour reconstruire les relations entre personnages, interpréter la réelle signification d'un divorce ou le comportement d'une personne lors d'un enterrement. On citera évidemment R. HOGGART, La culture du pauvre, Paris, Éd. de Minuit, 1970 ou O. SchWARTZ, Le monde privé des ouvriers, Paris, P.U.F., 1990.

2 T. MODLESKI, Loving With a Vengeance. Mass-Produced Fantasies for Women, Hamden, CT, Archon Books, 1982.

3 On notera que ces deux caractéristiques sont communes aux soaps et aux reality shows. Elles participent de ce qu'on appelle la néo-télévision, ou encore la télévision relationnelle. (D. MEHL, La fenêtre et le miroir, Paris, Payot, 1992). 
des points de vue, l'impossibilité de quelque solution définitive que ce soit, d'une régularisation de la situation. Bref, ce qu'offrent à leur public féminin les soaps, ce sont des problèmes complexes mais relevant de ses compétences, des situations auxquelles les spectatrices peuvent s'identifier -et ce, plus que des personnages, l'identification, selon le principe de la "mère idéale", étant toujours multiple et paradoxale. Ce que suggèrent ces approches féministes, c'est que le sens des soaps ne peut être compris qu'en référence à cet univers bien particulier qu'est la vie quotidienne des femmes au foyer. Il ne nous appartient pas ici d'évaluer la justesse de la description qu'en entreprend Modleski. Pour nous, l'intérêt de son approche est de montrer à quel genre de thématique, de problématique existentielle s'accrochent ces séries. Il nous parâtt intéressant d'articuler cette problématique autour du thème de l'ennui, thème qui nous permettra également de donner une cohérence aux trois questions que nous avons soulevées plus haut.

Une image semble résumer l'approche de Modleski : celle de la mère de famille seule dans sa maison, pendant que son mari est au travail et ses enfants à l'école. Et, sans doute, il s'agit bien du profil que visent de façon privilégiée les soaps, diffusés les après-midis des jours de semaine. Cependant, cette manière d'aborder le problème nous semble confiner trop le champ de pertinence du genre ; nous l'avons annoncé dès le départ, pour nous, la valeur des soaps réside dans leur potentiel métaphorique. Or, pour nous, le "blues de la ménagère" a bel et bien une valeur métaphorique. Notre hypothèse sera que ce à quoi la femme au foyer doit faire face, c'est la dislocation de son expérience. À propos du discours d'une ménagère qui s'ennuie, A. Wallemacq écrit : "d'une certaine façon, comme il n'y a plus de repères qui permettraient de déshomogénéiser le temps, il n'y a plus non plus de «moments» qui prendraient sens dans leurs différences réciproques. Il y a une indifférenciation de la journée, elle ne peut plus distinguer des moments dont la définition aurait une quelconque prégnance. (...) Lorsqu'elle décrit une journée (...), la phrase (peu ordonnée comme phrase) se perd dans une simple énumération dont les éléments sont à peine audibles"'. Nous avons eu l'occasion de vérifier, lors d'une recherche sur des jeunes femmes en situation de précarité, que l'ennui est une menace constante, même si

1 A. WallemacQ, L'ennui et l'agitation. Figures du temps, Bruxelles, De Boeck, 1991, p. 20-21 (italiques retirées). 
le travail à domicile est plus que suffisant ${ }^{1}$. Nous allons montrer maintenant que cette problématique de l'ennui déborde largement le cadre du public de soaps, et qu'elle est une catégorie centrale d'appréhension du monde contemporain.

\section{3. "Quelque chose s'est passé aujourd'hui"}

Jusqu'ici, nous n'avons fait que présenter ce qui a été dit, tout en cherchant à montrer comment les études les mieux connues "pressentent" ce que nous allons maintenant analyser avec plus de précision : qu'il n'est plus possible, aujourd'hui, de concevoir la télévision comme un instrument "de socialisation" dont on se servirait de l'extérieur pour "libérer" ou "aliéner" les "masses", mais qu'il faut replacer l'expérience concrète de "regarder la télévision" comme une partie à part entière de la vie -au même titre, par exemple, que "discuter avec des amis", "préparer le repas de midi" ou "dormir". Nous pourrons ainsi constater que les hypothèses baudrillardiennes relatives au simulacre et à l'effacement de la frontière entre le "réel" et le "virtuel" ne se rapportent pas seulement aux univers high-tech créés à grand frais et pour une population spécifique, mais également à des formes moins spectaculaires, plus quotidiennes.

\subsection{La nécessité de dépasser la sociologie critique sans pour autant l'abandonner}

Ce qui, d'après nous, se manifeste dans la prolifération dans la sociologie contemporaine de termes "phénoménologiques" -qu'on pense, par exemple, à la notion de rage ou de galère qu'utilise F. Dubet, au concept de souffrance de L. Boltanski, à celui d'incertitude d'A. Ehrenberg ou encore à la ruse dont parle M. de Certeau-, c'est une prise de conscience du fait que le sens des logiques sociales ne peut être approché que si on en ancre la compréhension dans l'expérience qui en est faite. Plus précisément, ces réflexions que les anciens sociologues pourraient juger esthétisantes et dès lors bourgeoises, nous semblent résulter d'une remise en question de la

1 E. Belin, Th.-M. Bouchat, Ph. Cornil \& B. Delvaux, Le sens au quotidien. Recherche avec des jeunes femmes en situation précaire, Bruxelles, Services Fédéraux des Affaires Scientifiques, Techniques et Culturelles, 1995. 
pertinence de la notion phénoménologique centrale de la sociologie critique : la notion d'aliénation, telle qu'elle s'est étoffée de Hegel à $H$. Arendt et aux théoriciens de l'école de Francfort. En matière de production culturelle, celle qui nous concerne ici, rien n'est plus significatif que la critique adornienne de la culture de masse, critique dont il est impossible de ne pas noter qu'elle se dit depuis un lieu dominant culturellement, et qu'elle consiste à stigmatiser, fût-ce pour la bonne cause, la conception de la vie d'un autre lieu, dominé celuilà, à partir de critères qui lui sont hétérogènes -pour dire les choses en bref : qu'elle est elle même méprisante et aliénante ${ }^{1}$. Le concept d'aliénation a eu, et possède encore dans certaines problématiques, une force d'évidence aujourd'hui perdue ; derrière ce que nous appelons les "nouveaux concepts sociologiques", ce qui se joue, nous semble-t-il, c'est la formulation d'une théorie critique susceptible d'avoir une efficacité sociale, mais qui repose sur des notions plus proches de l'expérience quotidienne contemporaine ${ }^{2}$.

L'ennui, explique Nahoum-Grappe, est avant tout le sentiment de perdre son temps, de vivre en marge de la vraie vie. "[L]'ennui est une faute vis-à-vis de soi et du don de la vie, il en est le refus lâche, pire qu'un échec, il fait passer «à côté de la vie». L'ennui est une faute non pas dans l'ordre de la morale et du devoir, mais dans celui de l'être et de l'existence. Un gâchis, une chance perdue, une occasion manquée. L'ennui produit la haine de soi après avoir détruit autour la consistance du monde, son éclat et sa frâ̂cheur violente"3. Ce sentiment d'être en faute, d'être indigne, le relie à la frustration quotidienne, entretenue par les promesses de la culture -promesses de couleurs, de plages, de boissons pétillantes, de signification. "Toute une imagerie de l'ennui comme signal d'une vie «fausse» désigne un programme de «la vraie vie»: (...) «se trouver soi-même, être soimême», but énigmatique qui se concentre sur ce point aveugle qu'est

1 Nous ne pouvons évidemment pas aborder ici ce débat épistémologique. Nous nous référons, pour notre part, à la réflexion menée par Z. BAUMAN, Legislators and Interpreters. On modernity, post-modernity and intellectuals, Cambridge, Polity Press, 1987 ; Z. BAuman, "Is There a Postmodern Sociology" in Theory, Culture and Society, vol 5 (1988), pp. 217-37. Nous élaborons notre position dans E. BELIN, "Bravades et bavardise: quel statut pour le discours sociologique?", Recherches Sociologiques, vol. XXVII, $\mathrm{n}^{\circ} 3$ (1996), p. 85-98.

2 Pour nous, l'horizon normatif qui se dessine lorsqu' on utilise des concepts comme celui d" "ennui" est celui de la barbarie tel que la conceptualise M. Henry, La barbarie, Paris, Grasset, 1987.

3 V. NAHOUM-GraPPE, L'ennui ordinaire, Paris, Aléas, 1995, p. 72. 
le «soi»"1. M. Henry développe une approche qui ne contredit pas cette description: "Dans l'ennui, suggère-t-il, à chaque instant une force se lève, se gonfle d'elle-même, se tenant prête, disposée à l'usage qu'on voudrait en faire. Mais que faire? «Je ne sais pas quoi faire». Aucune des voies hautes tracées par la culture et donnant licence à cette force de s'employer, à l'énergie de se déployer, à la vie de s'accroître elle-même et d'accomplir son essence-aucune de ces voies ne se présente à l'ennui pour que, s'engageant en elle dans un faire donc et dans l'épreuve de son souffrir, il se décharge de ce que l'inaction a d'oppressant"'?

Pour Henry la télévision est la figure même de l'ennui. La raison nous en semble simple : qu'il ne sait pas ce qu'est regarder la télévision. Nahoum-Grappe le souligne bien : l'ennui est de toutes les époques, de tous les milieux ; pourtant, il ne débouche pas forcément sur la barbarie ou, en tous cas, sur le fascisme. C'est ici que se dévoile ce qui, dans la vision moderne de l'émancipation, relève de la folie pure. Une seule chose semble à faire pour le révolutionnaire : I'histoire. Ainsi, chez Castoriadis : "Une véritable libération des énergies (...) passe par la marginalisation de tous les partis politiques existants, la création par le peuple de nouvelles formes d'organisation politique, fondées sur la démocratie, la participation de tous, la responsabilité de chacun à l'égard des affaires communes -bref, par la renaissance d'une véritable pensée et passion politique"3. À l'ennui, les modernes n'ont à proposer comme solution que l'histoire politique. Or l'ennui est ordinaire. Il est bien, pour parler comme Henry, ce par quoi la vie se déploie comme volonté de ne pas vivre, mais en cela, il est une de ces attitudes dans laquelle la vie se déploie, et on ne peut l'en dépouiller. Ou, pour le dire encore autrement, vouloir supprimer le risque de la barbarie, c'est vouloir amputer la vie d'une de ses dimensions, ce qui est barbare. "Nous avons toujours été des fans, disent Harrington et Bielby, parce que nous ne pouvons pas exister dans «un monde où rien n'a d'importance». Mais alors que les fans se sont traditionnellement impliqués dans des choses comme la politique, ou la religion, ou le travail, ils s'engagent aujourd'hui de

\footnotetext{
Ibid., p. 193.

M. HENRY, La barbarie, op. cit., p. 191.

3 C. CASTORIADIS, Domaines de l'Homme. Les carrefours du labyrinthe II, Paris, Éd. du Seuil, 1986, p. 117.
} 
manière privilégiée dans la culture populaire"1. Pour nous, ce genre de simplifications mérite plus que de méprisants ricanements.

\subsection{L'histoire transitionnelle et la reconstruction de l'expérience}

Travaux, de G. Navel, est l'autobiographie d'un ouvrier d'avantguerre. On l'y suit de chantiers en chaînes de montage, de mines de sel en vendanges, on le voit "transformer l'enfer en travail habitable"2. Puis, vers la fin, on lit: "Il m'apparaissait que l'ennui m'avait partout rejoint dans des conditions diverses de l'existence, dans les usines et sur les chantiers, avec ou sans Anna, et que partout où j'irais, ce n'était pas la peine de fuir, il me suivrait dans l'activité au travail ou dans les loisirs forcés; que l'ennui, l'état de sécheresse intérieure, encore plus que la faim, est le vrai mal des hommes ; qu'au travail, sauf dans les durs métiers du feu, la souffrance n'est pas la douleur musculaire, mais l'ennui (...). L'ennui est partout, à moins d'être amoureux, philosophe, savant, artiste, homme d'action ou d'affaires, paysan passionné de sa terre et de ses sous"3. Quelque pages plus loin, on le retrouve seul en train de recoudre un pantalon : "Je cousais à gros points. L'aiguille ne me demandait qu'un effort léger des doigts. Confondu avec cette occupation calme, je m'étais réveillé en train de vivre le temps comme le vivent les femmes dans leur monde intérieur quand elles sont seules et qu'elles cousent. J'avais songé à leur amour du beau, leur paix profonde et sans questions. Je songeais à celles qui fleurissent les églises, ornent leur maison, font de la dentelle, habillent au mieux leurs enfants, à leur délicatesse, à leur culte de la beauté"4. De fait, contre l'ennui, le remède, c'est la couture, la mise en ordre -au propre comme au figuré ! Une des jeunes femmes que nous avons interrogées nous expliquait sa passion pour les travaux ménagers: mettre de l'ordre autour d'elle, c'est mettre de l'ordre en elle 5 . Si nous avons cité longuement Navel, c'est parce que son texte illustre bien le caractère métaphorique de l'activité ménagère comme pointe vers une dimension fondamentale de l'existence.

1 C. L. HARRINGTON \& D. D. Bielby, Soap Fans... op. cit., p. 152-153.

2 G. NAvel, Travaux, Paris, Folio, 1945, p. 147.

3 Ibid., p. 205.

4 bid., p. 210.

5 Le sens au quotidien, op. cit., p. 67-69. 
Gardons à l'esprit la description du romancier, et considérons à présent la notion de "flow" dont nous avons parlé plus haut, et qui est le concept central de Csikszentmihalyi : "En état de flow, les actions se suivent les unes les autres selon une logique interne qui ne semble pas nécessiter d'intervention consciente de l'acteur. Celui-ci les expérimente comme l'écoulement d'un moment vers le suivant, dans lequel il contrôle ses actions, et où il y a peu de distinction entre le self et l'environnement, entre le stimulus et la réponse, ou entre le passé, le présent et le futur. Le flow est ce que nous appelons l'«expérience autotélique» (n.t.)"l. Pour Csikszentmihalyi, le flow n'est pas une expérience ordinaire : "dans la vie quotidienne, affirmet-il, les défis et les compétences sont rarement équilibrés. Soit qu'il y ait trop de choses à faire, qui exigent l'attention, auquel cas nous avons tendance à être soucieux ou anxieux ; soit qu'on ait l'impression de ne rien avoir à faire, auquel cas nous finissons par nous ennuyer. C'est pour cette raison que le flow se produit le plus souvent dans des activités structurées dans lesquelles le niveau de défis et de compétences peut être manipulé et contrôlé, comme par exemple les événements rituels, les jeux, les sports ou les performances artistiques (n.t.) $)^{2}$ Cependant, pour F. Massimini et M. Carli, "le flot des expériences ordinaires, qui s'étend du «doucement plaisant» à l'ennuyeux ou à l'angoissant, est fait d'un ensemble aléatoire de notes discordantes. Parfois, les notes se mettent en un accord harmonieux, et nous faisons l'expérience du flow (n.t.)"3. Nous l'avons annoncé plus haut, le sens de l'investissement dans les soaps réside dans la possibilité qui est offerte aux spectateurs ordinaires de circuler sur ce continuum qui va de ce que nous appelons la détente (Massimini et Carli utilisent le terme plus connoté d' "apathie") à l'excitation (ce qu'ils appellent flow).

L'étude du soap comme genre, au sens large que nous avons défini en début d'article, nous permet d'y découvrir un dispositif textuel, intertextuel et contextuel, par lequel sont précisément rendues manipulables les compétences mobilisables aussi bien que les sollici-

1 M. CSIKSZENTMIHALYI, Beyond Boredom and Anxiety, op. cit., p. 36.

2 M. Csikszentmihalyi \& I. S. CsikszentmiHalyi, Optimal experience. Psychological studies of flow in consciousness, Cambridge, Cambridge University Press, 1988, p. 31.

3 F. MASSIMINI \& M. CARLI, "The systematic assessment of flow in daily experience" in M. CSIKSZENTMIHALYi \& I. S. CsikszentMiHalyi, Optimal experience., op. cit., p. 267 (notre traduction). 
tations retenues comme pertinentes. Augmentation des compétences mobilisables, par exemple, dans l'acculturation progressive à l'histoire d'une série particulière, dans l'usage des textes secondaires et les échanges d'informations, dans la focalisation du texte sur des thématiques où les spectatrices disposent d'une compétence inutilisée dans leur vie quotidienne; filtrage des sollicitations dans l'exclusion de certaines thématiques comme celle du travail ou de la politique, dans l'utilisation de segments narratifs et de stéréotypes de présentation du réel, dans la sécurité de l'interaction télévisuelle. De ce point de vue, l'exaspération que suscitent ces textes chez les intellectuels apparaît à la fois comme une négation des compétences qui sont mises en œuvre dans l'expérience qui en est faite, et comme l'interdiction d'opérer sur le réel les réductions qui seules le rendent supportable. On débouche de la sorte sur la faiblesse de l'approche en terme de flow, qui est la pétition de principe sur le caractère indispensable de cette expérience dans la vie individuelle comme sociale. L'absence de réflexion sur la forme phénoménologique que prend l'adaptation cognitive nous semble être à l'origine d'une conception à notre sens trop métaphysique de la signification de ces expériences dans l'existence. Pour nous, les données de la psychologie cognitive ne sont pas erronées, mais elles sont insuffisantes lorsqu'on cherche à articuler les résultats opératoires à une sociologie compréhensive. En ce qui nous concerne, nous avons trouvé dans la théorie de l'espace potentiel de D.W. Winnicott des concepts qui, sans les infirmer, dépassent de loin sur le plan de la signification sociologique les observations de Csikszentmihalyi.

Reprenons quelques phrases de Nahoum-Grappe: "l'ennuyé regarde sans voir ou l'inverse, puisque une même tonalité a dévoré les contrastes et les éclats différenciés, puisque la grisaille a envahi le monde, puisque la pesée du vide l'immobilise et ralentit tous ses gestes"'. "Le sujet qui s'ennuie se sent terne, il n'est pas «brillant»"2. "Le bruit général du monde s'est tu autour des objets dont l'inertie atone met en échec la conscience. Elle perd alors sa tension verticale pour s'effondrer vers le bas"3. Ce lexique, D.W. Winnicott en répertorie l'exact inverse dans l'étude de ce qu'il appelle, suivant M. Klein, la défense maniaque: "remplir", "en mouvement", "coloré", "léger",

\footnotetext{
1 Op. cit., p. 63.

2 Ibid., p. 64.

3 lbid., p. 67.
} 
"s'élever", "ordre, harmonie", "comique" sont, explique-t-il, des termes fréquemment utilisés par des patients qui adoptent ce mode de défense contre la position dépressive ${ }^{1}$. La défense maniaque est caractérisée par une "fuite vers la réalité" consécutive à l'angoisse que provoque la découverte, à l'intérieur de soi, de mauvais objets, d'objets de mort. "Que dire du poste de radio qui fonctionne sans arrêt ? demande-t-il alors. De la vie dans une ville comme Londres avec son bruit incessant, ses lumières jamais éteintes ? Ce sont là des exemples de la réalité qui rassure vis-à-vis de la mort intérieure, et d'une utilisation de la défense maniaque qui peut être normale"2. Derrière cette phrase anodine se cache une approche de la culture que le psychanalyste développera tout au long de sa vie. Il n'est évidemment pas possible d'approfondir la question ici ; notons seulement que le "cercle des choses obscurcies" dont parle Nahoum-Grappe, c'est la trahison d'un monde qui ne nous rassure pas sur la mort intérieure, qui au contraire la reflète. La culture, dit Winnicott, est une forme "normale" de défense maniaque, c'est la "musique du monde", harmonieuse, rassurante, l'affirmation continuelle de la vraisemblance de la vie et de la victoire contre la mort. C'est, poursuit-il, l'essence de la mondanité : point n'est besoin de répondre aux angoisses, il suffit de se laisser porter par la réalité raffinée et aventureuse qui nous entoure.

Lorsque Winnicott parle de développement affectif, il utilise un concept qui, en réalité, dépasse de loin la traditionnelle opposition entre l'affectif et le cognitif. Ce qui se joue, dans l'équilibre dont parle Csikszentmihalyi, c'est bien plus qu'une équation "défiscompétences" : c'est l'évidence de la bienveillance du monde, la garantie qu'il est possible de vivre dans un environnement qui est à notre échelle. Ce n'est pas pour rien qu'une des études les plus riches dans le domaine du "flow" se rapporte à l'alpinisme : de fait, ce qui est en jeu dans ces expériences est de l'ordre du vertige, de l'attrait du vide. Les genres, de ce point de vue, apparaissent comme des dispositifs destinés à renverser les maximums de vraisemblance, c'est-à-dire des mondes bienveillants qui viennent s'interposer entre le monde hostile et le sujet, et à ce titre, reproduire avec les ressources du langage, de l'image et de tout un arsenal technique et économique, ce

1 D. W. WinnicotT, "La défense maniaque", in De la pédiatrie à la psychanalyse, Paris, Payot, 1958 (1935), p. 25.

2 Ibid., p. 21. 
geste dont la répétition monotone a rendu possible l'advention du sujet, celui par lequel un univers hostile est tenu à l'écart. Ce que Winnicott explique, en effet, c'est comment l'Edipe, le nouage de l'imaginaire et du symbolique, est simplement possible. Son génie a été de montrer qu'il est logiquement impossible, insupportable ; et que s'il est pratiquement réalisé, c'est grâce à un "mensonge". Ce mensonge est celui de la mère qui "prépare" l'enfant à cet affront suprême qu'il devra subir, en lui permettant, par sa prévenance durant les premiers instants, de croire dans l'amicalité du monde à son égard. Encore les mots nous piègent-ils ici : il n'y a pas de "monde" ni d'"égard", ni même de "mère" dans l'expérience des premiers jours. Il y a seulement l'expérience de la bienveillance. Winnicott, pour décrire la mère qui, par ses soins et sa disponibilité, dispense l'enfant de fréquenter le chaos, parle de "mère suffisamment bonne". Il indique par là qu'elle n'est jamais complètement bonne, mais qu'elle l'est suffisamment pour que cela ne soit pas traumatisant. C'est cet écart qu'il appellera, à la fin de sa vie, l'espace potentiel ${ }^{1}$. Cet espace ne se crée que par l'intervention explicite et constante d'un adulte, la mère, c'est-à-dire d'un autre qui l'illusionne sciemment et ne le désillusionne que par incapacité à l'illusionner totalement.

Mettre en place un monde à notre mesure, un univers qui se comporte selon nos désirs, comme s'il les prévenait, est une attitude qui débouche forcément sur la dissociation -mais tout groupe est dissocié, tout groupe est à «personnalités multiples». Lorsque la dupe ne trouve plus d'imposteur à sa mesure, qui la connaisse ce qu'il faut pour la séduire, et la domine assez pour qu'elle tombe sans retenue dans sa délicieuse escroquerie, elle ne peut plus compter que sur cette étrange opacité entre les innombrables «dedans» du monde de la vie. Cette «matière sombre» pétrie par la circulation des corps se cave parci, se découpe en crêtes par-là : des récifs se sculptent progressivement, et des ornières la strient. Tout cela compose un paysage dont les

1 "La mère s'adapte aux besoins de son bébé, de son enfant, tout au long de l'évolution progressive de sa personnalité et de son caractère. Cette adaptation lui confère un certain degré de fiabilité. L'expérience que fait le bébé de cette fiabilité pendant une période donnée suscite chez le bébé et l'enfant qui grandit un sentiment de confiance. C'est la confiance du bébé dans la fiabilité de la mère et, à partir de là, dans celle d'autres personnes et d'autres choses qui rend possible le mouvement de séparation entre le moi et le non-moi. Dans le même temps, cependant, on peut dire que la séparation est évitée, grâce à l'espace potentiel qui se trouve rempli par le jeu créatif, l'utilisation des symboles et par tout ce qui finira par constituer la vie culturelle" (D. W. WINNICOTT, Jeu et réalité, op. cit., p. 151. 
reliefs sont connus de tous, servent de points de repère dans les conversations; l'histoire s'écoulant, des édifices sémantiques se dressent sur le plan d'une cité commune : un soap opera est habité. Sans doute, la cuisine ou le salon appartiennent-ils à la maison de la ménagère, à son histoire tranquille, toujours proche de l'ennui, et peut-être le mari et les enfants sont-ils, en apparence, des maris et des enfants comme les autres; mais le sas de l'écran de télévision inscrit tous ces personnages, ces lieux, ces événements dans un autre plan, perpendiculaire. Ce que nous observons, c'est que ce monde sécant ne peut pas être si facilement déclaré fictif ou vaporeux, et ceci pour deux raisons complémentaires : d'une part, comme nous l'avons vu, ce que nous appelons le monde réel, à quoi nous conférons un statut de réalité privilégié, ce que nous décrétons être l'histoire, le travail, le plaisir, ne prend place que dans l'espace potentiel -le «monde où nous vivons»; mais il existe une seconde raison : que ce qui se déroule au sein du monde apparemment chimérique de l'expérience télévisuelle n'a rien d'ineffable ni de bénin : s'y mettent en place des systèmes complexes de cognitions, de sanctions, de récompenses, de souffrances et de jouissances qui n'ont rien de pâle comparés aux tracas et aux satisfactions que nous offre le «vraie» vie. Comme nous le mentionnions plus haut, les choses les plus sérieuses se passent lorsque nous jouons ; pour prendre un autre exemple, nous savons très bien que les douleurs les plus atroces, les angoisses les plus profondes, et même les états de conscience les plus lucides sur l'importance de ce qui est en train de se passer nous affectent souvent lorsque nous dormons, en rêve, alors même que, physiquement, et de manière paradigmatique, rien ne semble se passer. Nous voulons en venir à ceci : qu'au sein de cet univers enclavé dans l'espace domestique où rien ne se passe, se passent des choses d'une importance extrême, et que la production de cette importance, de ces enjeux, est d'une importance cruciale dans une société à ce point complexe que, pour beaucoup, elle en devient vertigineuse. 


\section{Conclusion}

Un terme nous semble décrire ce qu'apportent les "expériences génériques" de ce type dans la vie des spectateurs : de l'enjeu. Autour de ces textes se construit un univers, un monde parallèle au monde politique inaccessible et au monde quotidien qui se disloque. Ce monde est un des seuls où les spectatrices ont la possibilité d'être sujets. La question de savoir si elles le sont "vraiment" est une question insensée. La poser, c'est adopter la position du briseur de jeu ; or, à maints égards, cette position, c'est bien celle du critique, dont la fonction est de "sortir de l'illusion" en faisant "la part des choses". La question que nous soulevons ici n'est pas seulement éthique, elle est aussi logique : quel est le sens de tenir un discours désillusionnant à propos d'un ordre de réalité entièrement conçu autour de l'idée d'illusion. 